\title{
Avian Influenza A Virus Infection in Humans
}

\author{
Guang-Wu Chen ${ }^{1,2}$, Shin-Ru Shih ${ }^{1,3,4}$
}

$\mathrm{I}_{\mathrm{r} \text { r }}^{\mathrm{n}}$ nfluenza is a highly contagious, acute, and febrile respiratory illness that occurs in epidemics every winter and occasionally in pandemics. The disease is caused by the influenza virus, which belongs to the family Orthomyxoviridae. Influenza virions are enveloped particles containing a segmented, single-stranded RNA genome with negative polarity. ${ }^{[1]}$ The virus can be classified as influenza virus A or B, and influenza A virus can be further classified into subtypes based on the antigenic properties of the glycoproteins hemagglutinin (HA) and neuraminidase (NA). Thus far, 18 HA subtypes (H1-H18) and 11 NA subtypes (N1-N11) have been discovered ${ }^{[2]}$ Nearly all combinations of HA and NA subtypes circulate in avian species, except H17N10 and H18N11, which were recently identified in bats - the only mammal that can fly.

Avian species have been considered a reservoir for the influenza virus. Low pathogenic avian influenza (LPAI) viruses widely circulate in birds living near wetlands and aquatic environments. Despite being infected, most of these birds remain asymptomatic of developing illness. Genetic reassortment - an exchange of gene segments among different subtypes of virus - occurs frequently in the avian reservoir. Bird migration is the primary cause of different avian influenza viruses transferring from one geographic region to another. Wild avian species can spread the viruses to domestic poultry. Although most avian infections are asymptomatic, several subtypes, such as H5 and H7, can evolve to become highly pathogenic avian influenza (HPAI) viruses in poultry.

It is generally known that a "host species barrier" exists between avian species and humans for influenza A virus, which restricts the virus to bind only to a species-specific cell receptor or prevents an efficient replication subject to cellular factors. ${ }^{[3]}$ This is the primary reason that the avian influenza A virus cannot infect humans easily. However, several outbreaks of HPAI infections in poultry have occurred in the past two decades, and direct transmission of avian influenza A viruses to humans has been reported. For example, the H5N1 outbreak in Hong Kong in 1997 resulted in the first human cases of avian influenza virus infection. ${ }^{[4]}$ Following that, H5N1 viruses spread throughout numerous Asian countries including Indonesia, China, Thailand, and Vietnam, as well as to other continents. From 2003 to 2013, 648 cases of human H5N1 virus infection, of which 384 were fatal, were reported to the World Health Organization by 15 countries. ${ }^{[5]}$ Most of the infected individuals were reported to have had a history of direct contact with avian species; however, a number of family clusters have raised concern regarding human-to-human transmission. Although $\mathrm{H} 5 \mathrm{~N} 1$, in its current form, still lacks the ability to transmit efficiently among humans, it continues to pose a threat of becoming a human virus because of its potential to evolve through genetic drift from accumulating point mutations and genetic shift from reassortment.

Not only HPAI alone can cause human infection; at the end of April 2013, an LPAI H7N9 outbreak in China resulted in 131 confirmed human infections including 44 fatalities (statistics from February 2013 to April 2013). This new virus emerged from a reassortment of at least three subtypes of avian influenza virus: Its HA was derived from $\mathrm{H} 7 \mathrm{~N} 3$, NA from $\mathrm{H} 7 \mathrm{~N} 9$, and six other internal genes from H9N2. ${ }^{[6]}$ Subsequently, only sporadic cases were reported (two infections each in May, July, and October, with one death in July). Whereas infected avian species exhibited only mild or no symptoms, this new H7N9 virus caused severe pneumonia and acute respiratory distress syndrome in many human cases. It is believed that this novel H7N9 virus harbors several mammalian adaptation markers that are associated with increased replication and transmission in mammals. This might explain why this LPAI virus can also infect humans. Despite the reported human-to-human transmission of $\mathrm{H7N9},{ }^{[7]}$ the disease is still currently categorized as a "nonsustained" as opposed to a "sustained" human infection. In other words, H7N9 is an avian influenza virus and infects humans only when an individual has direct contact with infected poultry. It is not

From the ${ }^{1}$ Research Center for Emerging Viral Infections, College of Medicine, Chang Gung University, Taoyuan, Taiwan; ${ }^{2}$ Department of Computer Science and Information Engineering, School of Electrical and Computer Engineering, College of Engineering, Chang Gung University, Taoyuan, Taiwan; ${ }^{3}$ Department of Medical Biotechnology and Laboratory Science, College of Medicine, Chang Gung University, Taoyuan, Taiwan; ${ }^{4}$ Clinical Virology Laboratory, Department of Clinical Pathology, Chang Gung Memorial Hospital at Linkou, Taiwan

Received: Jan. 08, 2014; Accepted: Jan. 20, 2014

Correspondence to: Prof. Shin-Ru Shih, Research Center for Emerging Viral Infections, College of Medicine, Chang Gung University. 259 Wenhua $1^{\text {st }}$ Rd., Gueishan, Taoyuan 333, Taiwan. Tel: 886-3-2118800 ext. 5497; Fax: 886-3-2118174; E-mail: srshih@ mail.cgu.edu.tw

DOI: $10.4103 / 2319-4170.125882$ 
capable of spreading easily among humans to be referred as "human influenza A virus."

In addition to $\mathrm{H} 5 \mathrm{~N} 1$ and $\mathrm{H} 7 \mathrm{~N} 9$, other avian influenza viruses have also been reported to infect humans. In 1999, avian influenza A H9N2 viruses were isolated from the nasopharyngeal aspirates of two children, and the illness was mild and self-limited in both cases. The H9N2 human isolates shared high sequence similarity with that of poultry isolates identified in the same year, ${ }^{[8]}$ and sporadic human cases have been reported since 1999. In 2003, an outbreak of the H7N7 virus in poultry in the Netherlands resulted in 89 human cases, including one death. ${ }^{[9]}$ More recently, the first-ever reported human case of avian H6N1 virus infection was confirmed in Taiwan. ${ }^{[10]}$ This case was a mild infection in a 20-year-old woman. The entire genome sequence of this virus was quickly released by the National Influenza Center, Center for Disease Control, Taiwan, and the segment-wise analysis indicated that several internal genes of this new virus originated from another $\mathrm{H} 5 \mathrm{~N} 2$ avian virus, which has been circulating in Taiwan for years. The virus exhibits a G228S substitution in the HA protein, which might affect its receptor-binding affinity. Although this is the only human case identified thus far, the frequent reassortment and high prevalence of $\mathrm{H} 6$ viruses in avian species have raised concerns about public health. Continued monitoring of $\mathrm{H} 6 \mathrm{vi}$ ruses in Taiwan and the surveillance of other avian influenza viruses in unknown-pathogen-pneumonia cases are critical.

\section{REFERENCES}

1. Lamb RA, Krug RM. Orthomyxoviridae: The viruses and their replication, in Fields virology, $4^{\text {th }}$ Edition. Knipe DM, Howley PM, Griffin DE, Lamb RA, Martin MA, Roizman B, et al. editors, Philadelphia, PA: Lippincott-Raven; 2001. p. 1487-531.

2. Tong S, Zhu X, Li Y, Shi M, Zhang J, Bourgeois M, et al. New World Bats Harbor Diverse Influenza A Viruses. PLoS Pathog 2013;9:e1003657.

3. Chen GW, Shih SR. Genomic signatures of pandemic (H1N1) 2009 influenza virus. Emerg Infect Dis 2009;15:1897-903.

4. Claas EC, Osterhaus AD, van Beek R, De Jong JC, Rimmelzwaan GF, Senne DA, et al. Human influenza A H5N1 virus related to a highly pathogenic avian influenza virus. Lancet 1998;351:472-7.

5. WHO website - Cumulative number of confirmed human cases of avian influenza A (H5N1) reported to WHO. Available from: http://www.who.int/influenza/human_animal_interface/H5N1 cumulative_table_archives/en/index.html. [Last accessed on $201 \overrightarrow{4}$ Jan 10 ].

6. Gao R, Cao B, Hu Y, Feng Z, Wang D, Hu W, et al. Human infection with a novel avian-origin influenza A (H7N9) Virus. N Engl J Med 2013;368:1888-97.

7. Qi X, Qian YH, Bao CJ, Guo XL, Cui LB, Tang FY, et al. Probable person to person transmission of novel avian influenza A (H7N9) virus in Eastern China, 2013: Epidemiological investigation. BMJ 2013;347:f4752

8. Peiris M, Yuen KY, Leung CW, Chan KH, Ip PL, Lai RW, et al. Human infection with influenza H9N2. Lancet 1999;354:916-7.

9. Fouchier RA, Schneeberger PM, Rozendaal FW, Broekman JM, Kemink SA, Munster V, et al. Avian influenza A virus (H7N7) associated with human conjunctivitis and a fatal case of acute respiratory distress syndrome. Proc Natl Acad Sci U S A 2004;101:1356-61.

10. Wei SH, Yang JR, Wu HS, Chang MC, Lin JS, Lin CY, et al. Human infection with avian influenza A H6N1 virus: An epidemiological analysis. Lancet Respir Med 2013;1:771-8. 